\title{
ON THE NORMALISER PROBLEM FOR G-ADAPTED GROUP RINGS OF TORSION GROUPS
}

\author{
YUANLIN Li
}

\begin{abstract}
In this note, we prove that if a torsion group $G$ has an Abelian subgroup $B$ such that $G / B$ is Abelian and $R$ is a $G$-adapted ring with the property that $R(G / B)$ has only trivial units then $G$ has the normaliser property in $R G$.
\end{abstract}

\section{Introduction and Preliminary}

Let $G$ be a torsion group. A ring $R$ is called a $G$-adapted ring if $R$ is an integral domain of characteristic 0 in which no prime dividing the order of any element of $G$ is invertible. Throughout this article the coefficient ring $R$ in the group ring $R G$-is always assumed to be a $G$-adapted ring. Let $\mathcal{U}(R G)$ be the group of units of the $G$-adapted group ring $R G$ and $N_{\mathcal{U}}(G)$ be the normaliser of $G$ in $\mathcal{U}(R G)$. Clearly, $N_{\mathcal{U}}(G)$ contains $G$ and also contains $\mathcal{Z}=\mathcal{Z}(\mathcal{U}(R G))$, the subgroup of central units of $\mathcal{U}$. In [15] Question 43 (that is, the normaliser problem) asks whether $N_{\mathcal{U}}(G)=G \mathcal{Z}$ when $G$ is finite. If the above equality holds, then we say that $G$ has the normaliser property in $R G$. It was believed that the equality holds for all finite groups until recently Hertweck found counterexamples to the problem. Using them and a smart generalisation of a result of Mazur [13], Hertweck constructed a counterexample to the well-known isomorphism problem [1]. Since the normaliser problem is intimately connected to the isomorphism problem it is very much of interest to know which groups enjoy the normaliser property. In the past few years, a great amount of work on the problem has been done by several authors (see $[2,3,6,8,9,10,11,12,14])$. In this paper we prove that if a torsion metabelian group $G$ has an Abelian subgroup $B$ such that $G / B$ is Abelian and $R(G / B)$ has only trivial units, then $G$ has the normaliser property in $R G$. This extends a result of [9, Proposition 2.20] on a finite group basis $G$ in its integral group ring, to any torsion group basis $G$ in its $G$-adapted ring.

Every unit $u \in N_{\mathcal{U}}(G)$ induces an automorphism $\varphi$ of $G$ such that $\varphi_{u}(g)=u g u^{-1}$ for all $g \in G$. We now consider the subgroup $\operatorname{Aut}_{\mathcal{U}}(G)$ formed by all such automorphisms

Received 12th August, 2002

This research was supported in part by a research grant from the Natural Sciences and Engineering Research Council of Canada.

Copyright Clearance Centre, Inc. Serial-fee code: 0004-9727/03 \$A2.00+0.00. 
and it is not hard to see that the normaliser problem described in [15] is equivalent to [5, Question 3.7]:

$$
\text { "Is } \operatorname{Aut}_{\mathcal{U}}(G)=\operatorname{Inn}(G) \text { for all finite groups } G \text { ?" }
$$

It is convenient to use this equivalent form to discuss the normaliser problem here and our notation follows that in [15].

Next we introduce some terminology and preliminary results.

Definition 1.1: Let $G$ be a torsion group. A subgroup $P$ is called a Sylow $p$ subgroup of $G$ for a prime number $p$, if $P$ is a maximal $p$-subgroup of $G$.

It is not hard to see that there exists a maximal $p$-subgroup of $G$ by Zorn's Lemma. We remark that the Sylow theorems for finite groups are no longer true in this context. For example, not all Sylow $p$-subgroups are conjugates of one another. We need the following two results. A proof for the first can be found in [7, 1.D.4. Lemma] and the second is a special case of [14, Theorem 9].

LEMMA 1.2. If $B$ is a normal subgroup of a locally finite group $G$ such that the quotient group $G / B$ is a countabie $p$-group for some prime $p$ then there is a $p$-subgroup $P$ of $G$ with $B P=G$.

LEMMA 1.3. Let $G$ be a torsion group and $P$ be any $p$-subgroup of $G$. For any $u \in N_{\mathcal{U}}(G)$, define $\varphi \in \operatorname{Aut}(G)$ such that $\varphi(g)=u g u^{-1}$ for every $g \in G$ as before. Then restricted to the subgroup $P$, the automorphism $\varphi$ becomes inner. Moreover, we have $\left.\varphi\right|_{P}=\left.\operatorname{conj}\left(x_{0}\right)\right|_{P}$ for some $x_{0} \in \operatorname{supp}(u) \subset G$. In particular, if $G$ is a $p$-group, then $\operatorname{Aut}_{\mathcal{U}}(G)=\operatorname{Inn}(G)$, so the normaliser property holds for $G$.

We include a proof for completeness, and we note that the technique used in the following proof will be required later in the proof of Lemma 2.2.

Proof: Let $u=\sum u(x) x \in N_{\mathcal{U}}(G)$, where $u(x) \in R$ and $x \in \operatorname{supp}(u)$. Without loss of generality, we may assume that the augmentation of $u$ is 1 . For every group element $g \in G, \varphi(g)=u g u^{-1}$ is also a group element. Rewrite $u=\varphi(g) u g^{-1}$, and hence $\sum u(x) x=\sum u(x) \varphi(g) x g^{-1}(*)$. This shows that $\varphi(g) x g^{-1}$ is in the support of $u$ for all $g \in G$. Define a left group action $\sigma_{g}$ of $G$ on $\operatorname{supp}(u)$ as follows: $\sigma_{g}(x)=\varphi(g) x g^{-1}$. It follows from $(*)$ that $u(x)$ is a constant on each orbit of $x$. Restricting the action to $P$, we have that the $p$-subgroup $P$ acts on $\operatorname{supp}(u)$, and thus the length of every orbit must be a $p$-power. It follows that

$$
1=\varepsilon(u)=\sum c_{i} p^{l_{i}}
$$

where $\varepsilon$ is the augmentation map, $p^{l_{i}}$ is the length of the orbit of $x_{i}$ and $u\left(x_{i}\right)=c_{i}$. Since $p$ is not invertible in $R$, the above equality shows that $p^{l_{j}}=1$ for some $j$; that is to say there is a fixed point of this action, say $x_{0}$. Therefore, we have $\varphi(g) x_{0} g^{-1}=\sigma_{g}\left(x_{0}\right)=x_{0}$ for all $g \in P$. Consequently, $\varphi(g)=x_{0} g x_{0}^{-1}$, and thus $\left.\varphi\right|_{p}=\left.\operatorname{conj}\left(x_{0}\right)\right|_{P}$. We are done. $\square$ 


\section{The Main Result}

In this section, we extend our earlier result on a finite group basis $G$ ([9, Proposition $2.20])$ to any torsion group basis $G$. The main result is as follows:

THEOREM 2.1. Let $G$ be a torsion metabelian group and let $B$ be an Abelian normal subgroup of $G$ for which the quotient group $A=G / B$ is $A$ belian. If $R A$ has only trivial units then $G$ has the normaliser property in $R G$.

We prove Theorem 2.1 by means of the following two lemmas.

LEMMA 2.2. Let $G$ be a torsion metabelian group and let $B$ be an $A$ belian normal subgroup of $G$ for which the quotient group $A=G / B$ is Abelian. Let $A_{p}$ be the Sylow p-subgroup of $A$ and $G_{p}$ be the pre-image of $A_{p}$ in $G$. If $R A$ has only trivial units, then for every $\varphi$ in $\operatorname{Aut}_{\mathcal{U}}(G)$ the restriction of $\varphi$ to $G_{p}$ is inner.

Proof: Let $u \in N_{\mathcal{U}}(G)$ and $\varepsilon(u)=1$. Define $\varphi$ to be the automorphism induced by $u$ as before. We shall show that the restriction of $\varphi$ to $G_{p}$ is inner.

First we show that the restriction of $\varphi$ to $B$ is inner. Write $u=\sum_{i=0}^{n} \alpha_{i} a_{i}$ where the cosets $a_{i} B$ are pairwise distinct, $a_{0}=1$, and $\alpha_{i} \in R B$. Since $R A$ has only trivial units, we have that in $R A, \bar{u}=\sum_{i=0}^{n} \varepsilon\left(\alpha_{i}\right) \overline{a_{i}}$ is trivial. Thus only one $\varepsilon\left(\alpha_{l}\right)=1$ and the others are zero. That is $\varepsilon\left(\alpha_{j}\right)=0$ for all $j \neq l$. Multiplying by $a_{l}^{-1} b^{-1}$ for any $b$ in the support of $\alpha_{l}$ if necessary, we may assume that $\varepsilon\left(\alpha_{0}\right)=1, \varepsilon\left(\alpha_{i}\right)=0$ for all $i \neq 0$ and $1 \in \operatorname{supp}(u)$. Now we show that $\varphi(b)=b$ for all $b \in B$ (or $\left.\varphi\right|_{B}=\left.i d\right|_{B}$ ). Since $B$ is a normal subgroup and $u \in N_{U}(G)$, we have that $[u, b]=u b u^{-1} b^{-1} \in G$. By going $\bmod B$, we obtain that $[\bar{u}, \bar{b}]=\overline{1}$, and therefore, $[u, b] \in B$. Thus there exists a $b_{0} \in B$ depending on $b$ such that $u b=b_{0} b u$. This implies that $\alpha_{0} b=b_{0} b \alpha_{0}$, and therefore, $\alpha_{0}\left(1-b_{0}\right)=0$. This simply says that the order of $b_{0}$ divides $\varepsilon\left(\alpha_{0}\right)=1$, forcing $b_{0}=1$. Hence $[u, b]=1$, and thus $\varphi(b)=b$ for all $b \in B$. We remark that because $1 \in \operatorname{supp}(u)$, the support of $u$ is contained in the $F C$-centre of $G$ by [14, Corollary 1]. Since $G$ is locally finite, it follows that the support of $u$ is contained in some finite normal subgroup $H$ of $G$. Therefore, the support of $\alpha_{0}$ is contained in the finite normal subgroup $H_{1}=B \cap H$.

Next let $P$ be any $p$-subgroup of $G$. We now show that the restriction of $\varphi$ to $P$ is a conjugation by a group element $b_{1}$, and moreover, $b_{1} \in \operatorname{supp}\left(\alpha_{0}\right) \subset B$. The first result follows from Lemma 1.3. To show the second part, we use the same trick as that used in the proof of Lemma 1.3. This time we need only define a group action of $P$ on the support of $\alpha_{0}$, and then the fixed point $b_{1} \in \operatorname{supp}\left(\alpha_{0}\right)$ will do the job. We observe that for every $p \in P, \varphi(p) p^{-1}=u p u^{-1} p^{-1} \in B$ because in $R A, \overline{\varphi(p) p^{-1}}=\overline{1}$. Since $u p=\varphi(p) u$ and $u=\sum_{i=0}^{n} \alpha_{i} a_{i}$, we have $\sum_{i=0}^{n} \alpha_{i} a_{i} p=\sum_{i=0}^{n} \varphi(p) \alpha_{i} a_{i}$. This shows that $\alpha_{0} p=\varphi(p) \alpha_{0}$ and thus $\alpha_{0}=\varphi(p) \alpha_{0} p^{-1}$. Write $\alpha_{0}=\sum \alpha_{0}(b) b$, where $b \in \operatorname{supp}\left(\alpha_{0}\right), \alpha_{0}(b) \in R$. It follows that $\sum \alpha_{0}(b) b=\sum \alpha_{0}(b) \varphi(p) b p^{-1}$. Now we can define a group action of $P$ on $\operatorname{supp}\left(\alpha_{0}\right)$, sending every element $b$ to $\varphi(p) b p^{-1}\left(\in \operatorname{supp}\left(\alpha_{0}\right)\right)$. As before, we can show that there 
is a fixed point $b_{1} \in \operatorname{supp}\left(\alpha_{0}\right)$. Therefore, $\varphi(p)=\operatorname{conj}\left(b_{1}\right)(p)$ for all $p \in P$. Since $b_{1} \in B$ and $B$ is Abelian, we conclude that $\left.\operatorname{conj}\left(b_{1}\right)\right|_{B}=\left.i d\right|_{B}=\left.\varphi\right|_{B}$, and therefore, $\left.\varphi\right|_{B P}=\left.\operatorname{conj}\left(b_{1}\right)\right|_{B P}$.

Finally we prove that the restriction of $\varphi$ to $G_{p}$ is a conjugation by a group element $b_{p}$ in the support of $\alpha_{0}$. For every finite subgroup $F$ of $G_{p}$, we can find a finite subgroup $A_{F}$ of $A_{p}$ such that $F$ is contained in the pre-image of $A_{F}$ in $G$. By Lemma 1.2, there exists a $p$-subgroup $P$ of $G$ such that this pre-image is equal to $B P$. It follows that there is a $b_{F}$ in the support of $\alpha_{0}$ such that $\varphi$ acts on $F$ as conjugation by $b_{F}$. Since the support of $\alpha_{0}$ is finite, it is not hard to see that one can find a $b_{p}$ which works for all $F$. In fact if this is not true, then for each $b$ in the support of $\alpha_{0}$ there is such a $F_{b}$ so that $\varphi$ is not a conjugation by $b$ on it. Therefore, for the finite subgroup generated by the groups $F_{b}$ for all $b \in \operatorname{supp}\left(\alpha_{0}\right)$, there would be no $b$ such that $\varphi$ is a conjugation by $b$ on it. This leads to a contradiction. Note that $G_{p}$ is the union of its finite subgroups, so the restriction of $\varphi$ to $G_{p}$ is a conjugation by $b_{p}$. Without loss of generality, for a fixed $p$ we may assume that $\left.\varphi\right|_{G_{p}}=\left.i d\right|_{G_{p}}$. We note that the support of $\alpha_{0}$ is still contained in $H_{1}$, and what we just proved implies that the order of $\varphi$ is finite, so we may assume that $\varphi$ has a prime power order in the sequel.

Lemma 2.3. Let $G$ be a torsion metabelian group and let $B$ be an Abelian normal subgroup of $G$ for which the quotient group $A=G / B$ is an Abelian group of exponent 6. If $R A$ has only trivial units then $G$ has the normaliser property in $R G$.

. Proof: Let $\varphi=\varphi_{u} \in \operatorname{Aut}_{\mathcal{U}}(G)$ where $u=\sum_{i=0}^{n} \alpha_{i} a_{i}$ as before. We now show that $\varphi$ is inner. Let $A=A_{2} \times A_{3}$, and let $G_{p}$ be the pre-image of $A_{p}$ in $G$ for $p=2$ or 3 as before. As we mentioned earlier, we may assume that the order of $\varphi$ is a power of $p$. If $p \neq 3$, by Lemma 2.2 we may further assume that $\left.\varphi\right|_{G_{2}}=\left.i d\right|_{G_{2}},\left.\varphi\right|_{G_{3}}=\left.\operatorname{conj}\left(b_{3}\right)\right|_{G_{3}}$ for some $b_{3} \in \operatorname{supp}\left(\alpha_{0}\right) \subset H_{1}$, where $H_{1}$ is the same subgroup as that used in the proof of Lemma 2.2. Now we define a map $\delta$ from $A$ to $B$ as follows: $\delta(a)=\varphi(g) g^{-1}$ for every $a \in A$, where $g$ is any pre-image of $a$ in $G$. It is routine to check $\delta$ is well defined, $\delta(a) \in B$, and $\delta(a)$ is a $1-$ cocycle. We claim that $\delta^{k}(a)=\varphi^{k}(g) g^{-1}$. For $k=1$, this is clear. By induction, we assume that $\delta^{k-1}(a)=\varphi^{k-1}(g) g^{-1}$. Then

$$
\delta^{k}(a)=\delta^{k-1}(a) \delta(a)=\varphi^{k-1}(g) g^{-1} \varphi(g) g^{-1}=\varphi\left(\varphi^{k-1}(g) g^{-1} g\right) g^{-1}=\varphi^{k}(g) g^{-1} .
$$

Since $o(\varphi)$, the order of $\varphi$, is a power of $p$ and $\delta^{o(\varphi)}(a)=\varphi^{o(\varphi)}(g) g^{-1}=1$, we conclude that the order of $[\delta]$ is a power of $p$. On the other hand, we shall show that the order of $[\delta]$ is a power of 3 . Therefore, $[\delta]$ is trivial. We first note that the restriction of $[\delta]$ to $A_{2}$ is trivial. This is because for all $a_{2} \in A_{2}, \delta\left(a_{2}\right)=\varphi\left(g_{2}\right) g_{2}^{-1}=1$, where $g_{2} \in G_{2}$ is any pre-image of $a_{2}$ in $G$, so $\varphi\left(g_{2}\right)=g_{2}$ by the above assumption. Since $H_{1}$ is a normal subgroup and $H_{1}$ is contained in the Abelian subgroup $B$, we can define a group action of $A_{3}$ on $H_{1}$ by conjugation (that is, for every $a_{3} \in A_{3}, h_{1}^{a_{3}}=h_{1}^{g_{3}} \forall h_{1} \in H_{1}$, where $g_{3}$ is 
any pre-image of $a_{3}$ in $G$ ). Because $H_{1}$ is finite, some subgroup $C$ of $A_{3}$ of finite index $k$ acts trivially on $H_{1}$. This means that the pre-image of $C$ in $G$ centralises $H_{1}$. It follows that for every $a_{3} \in C, \delta\left(a_{3}\right)=\varphi\left(g_{3}\right) g_{3}^{-1}=b_{3} g_{3} b_{3}^{-1} g_{3}^{-1}=1$, where $b_{3}$ is in the support of $\alpha_{0}\left(C H_{1}\right)$ as before. So the restriction of $[\delta]$ to $C$ is also trivial. Now write $A_{3}=C \times C^{\prime}$, where the order of $C^{\prime},\left|C^{\prime}\right|=k$, is a power of 3 . It is well known that the restriction of $\left[\delta^{k}\right]$ to $C^{\prime}$ is trivial. Thus $\left[\delta^{k}\right]$ is trivial, and then the order of $[\delta]$ is a power of 3 . Therefore, $[\delta]$ is trivial and thus, $\delta$ is a coboundary. Now we conclude that $\varphi$ is inner.

In the case that $p=3$, we may assume that $\left.\varphi\right|_{G_{3}}=\left.i d\right|_{G_{3}},\left.\varphi\right|_{G_{2}}=\left.\operatorname{conj}\left(b_{2}\right)\right|_{G_{2}}$ for some $b_{2} \in \operatorname{supp}\left(\alpha_{0}\right) \subset H_{1}$. Following the same line as that in the above, we can prove that $\varphi$ is inner. Therefore, this completes the proof.

Now we are ready to prove our main result (Theorem 2.1).

Since a $G$-adapted ring $R$ is an integral domain of characteristic 0 , we may assume that $\mathbb{Z}$ the ring of all rational integers is a subring of $R$. Because $R A$ has only trivial units, $\mathbb{Z} A$ has only trivial units too. So it follows from Higman's Theorem ([4], see also [15, Theorem 2.7]) that $A$ is an Abelian group of exponent $2,3,4$ or 6 . If $A=G / B$ is an Abelian group of exponent 2,4 or 3, then $G=G_{2}$ or $G=G_{3}$. Therefore, the result follows from Lemma 2.2. In the case that $A$ is an Abelian group of exponent 6 , the result follows from Lemma 2.3 .

\section{REFERENCES}

[1] M. Hertweck, 'A counterexample to the isomorphism problem for integral group rings', Ann. of Math. 154 (2001), 115-138.

[2] M. Hertweck, 'Class-preserving automorphisms of finite groups', J. Algebra 241 (2001), $1-26$.

[3] M. Hertweck and W. Kimmerle, 'Coleman automorphims of finite groups', Math. 2. 242 (2002), 203-215.

[4] G. Higman, 'The units of group rings', Proc. London Math. Soc. (2) 46 (1940), 231-248.

[5] S. Jackowski and Z. Marciniak, 'Group automorphisms inducing the identity map on cohomology', J. Pure Appl. Algebra 44 (1987), 241-250.

[6] E. Jespers, S.O. Juriaans, M. de Miranda and J.R. Rogerio, 'On the normalizer problem', J.Algebra 247 (2002), 24-36.

[7] O.H. Kegel and B.A.F. Wehrfritz, Locally finite groups (North-Holland, Amsterdam, London, 1973).

[8] W. Kimmerle, 'On the normalizer problem', in Algebra, Trends in Math. (Birkhauser, Basel, 1999), pp. 89-98.

[9] Y. Li, 'The normalizer of a metabelian group in its integral group ring', J. Algebra (2002) (to appear).

[10] Y. Li, M.M.Parmenter and S.K.Sehgal, 'On the normalizer property for integral group rings', Comm. Algebra 27 (1999), 4217-4223.

[11] Z.S. Marciniak and K.W. Roggenkamp, 'The normalizer of a finite group in its integral group ring and Čech cohomology', in Algebra - Representation Theory, 2001 (Kluwer Academic Publishers, 2001), pp. 159-188. 
[12] M. Mazur, 'Automorphisms of finite groups', Comm. Algebra 22 (1994), 6259-6271.

[13] M.Mazur, 'On the isomorphism problem for integral group rings of infinite groups', $E x$ position. Math. 13 (1995), 433-445.

[14] M.Mazur, 'The normalizer of a group in the unit group of its group ring', J. Algebra 212 (1999), 175-189.

[15] S.K. Sehgal, Units in integral group rings, Pitman Monographs in Pure and Applied Maths. 69 (Longman Scientific and Technical, Harlow, 1993).

Department of Mathematics

Brock University

500 Glenridge Ave.

St. Catharines, Ontario

LZS 3A1 Canada

e-mail: yli@brocku.ca 